\title{
Daylight Performances of Atrium Buildings for Different Roof Configuration Under Malaysia Sky Conditions
}

\author{
Julitta Yunus ${ }^{1, *}$, Sabarinah Sh Ahmad ${ }^{1}$ and Azni Zain-Ahmed ${ }^{1}$ \\ ${ }^{1}$ Faculty of Architecture, Planning and Surveying, Universiti Teknologi MARA (UiTM), 40450, Shah Alam, Selangor, Malaysia
}

\begin{abstract}
The research endeavours to fill the identified gap in the field's knowledge on daylighting in atriums, specifically under tropical sky conditions. The atrium's form, roof configurations, building orientation, roof transmittance, internal surfaces reflectivity, and grazing areas are the key elements in the daylighting design of atrium buildings. Daylight availability in an atrium has been researched extensively using different techniques and models. This paper presents substantial findings from a virtual simulation exploration. Four models were used to perform the daylighting performance analyses involving field experiments and computer simulations (IESRadiance). The models used different roof fenestration designs and structural truss systems (flat, pitched, pyramidal-gridded and saw-tooth roofs) simulated for substantial virtual verification and investigation. The Daylight Factor/Daylight Ratio (DF/DR) on horizontal surfaces under different types of sky conditions at different times of day and different days over a period of one year were obtained. The illuminance levels were quantified for the unique circumstances of intermediate sky conditions. They show a generally linear relationship and a good degree of correlation with the overall reduction of daylight levels in the atrium. Internal roof obstructions created a relatively constant attenuation of daylight compared with the clear unobstructed roof for the four structured roofs. The discrepancy of light distribution was increased if measured between the unobstructed atrium well with complex configurations. This work has paved the way for more design strategies using complicated atrium roof fenestration constructions to be analysed with accuracy while combining aesthetics with energy efficiency.
\end{abstract}

\section{Introduction}

The aim of this paper is to investigate the effects of typical modern atrium roofs configurations upon daylight transmittance for atrium buildings in Malaysia. Three types of atrium roof configurations; flat, pitched, and pyramidal-gridded roof structures were used to predict the daylight performance in atrium buildings.

The extensive conclusion from the exploration of the daylighting performance of an atrium is one complex issue [1], not to mention with the inclusion of atrium roof configurations and tropical sky conditions. These elements were critically reviewed and the directive is towards improving the daylight strategies with different passive applications. To demonstrate with more accurate results, the author further investigates and analyse the effects of several variations in the roof structure and other atrium configurations on the daylight levels. This was made specifically for atrium buildings under the tropical sky conditions of Malaysia. The exploration is enhanced with a series of investigations and virtual experiment evaluations.

This research began with the exploration of daylighting in the atrium. Initially, a typological study on existing atrium form, shape, and atrium roof configurations in Malaysia was conducted. The survey done by Yunus [2] was to determine the physical characteristics of a typical atrium building and roof structure for the tropical climate. It was found that the preferred design of atrium under tropical regions is the top-lit and enclosed rectangular shallow atrium with an average height of four storeys and well index of one $(\mathrm{WI}=1)$ as verified in [3] and supported by other works [4-6]. A field study was then conducted using scale models under real sky conditions as reported in [2]. From this, three atrium roof models (structured flat, pyramidal-gridded and pitched roof) that reflected typical tropical atrium were developed to investigate the daylight performances. The findings from the field study were then further used in the virtual simulation to predict the daylight performance of atrium buildings under tropical sky conditions.

\section{Methodology}

This section describes the investigation of remodelled typical atrium buildings with the inclusion of different roof structure affects daylight levels in atrium under tropical sky conditions using virtual model simulations. The four-sided, top-lit, square-shaped atrium well base remained the same and experimental procedures were repeated with roof variants accordingly. The best and worst case scenarios were applied and further

\footnotetext{
Corresponding author: author@e-mail.org
} 
investigated. Then, possible design solutions were formulated and verified in terms of daylight performance under more complex conditions and time variances using the IESRadiance simulation tools.

\subsection{Modelling, positioning and setting up}

With ModelIT, a full-scale virtual model was developed with three (3) different roof fenestration designs. Fig. 1 shows one example of pitched-roof developed with ModelIT positioned in the model viewer. The impact of all the types of roof fenestration systems on daylight level and illuminance distribution was examined at the centre, central edges and corner positions as shown in Fig. 2 on the atrium floor at different orientations (North, South, East and West).

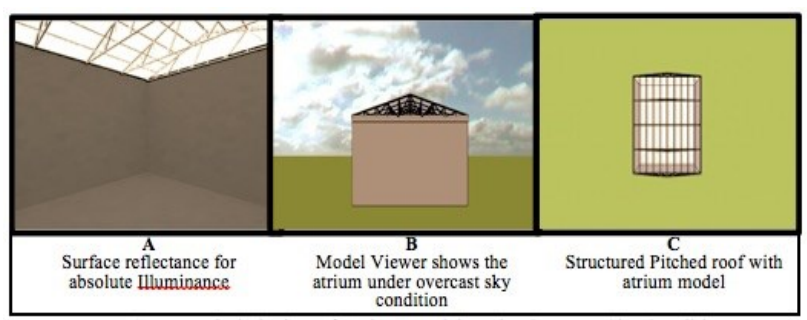

Fig. 1. Structured Pitched-roof atrium modelled with ModelIT viewed in Model Viewer

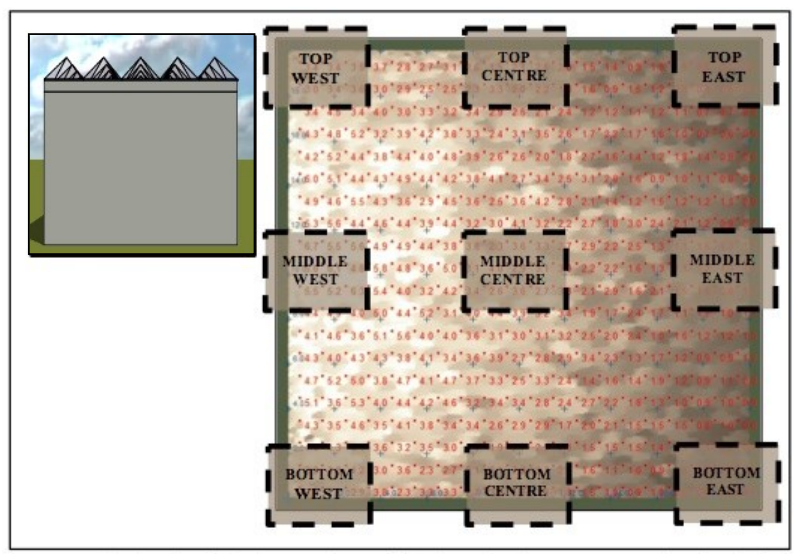

Fig. 2. Illuminance grid on atrium floor with measurement of main points positions.

\subsection{Illuminances measurement, sky conditions and simulation}

The sky types covering Malaysia are namely the Commission Internationale de 'Eclairage (CIE) standard overcast sky, intermediate with sun and clear sky which is $83 \%$ of the total frequency of occurrence. Two out of the three types were used in the simulation study of daylight performance in the atrium. The IESRadiance module of the software was used to simulate the illuminance and DF in the atrium. Mousavi [7] had validated the software especially for the DF and daylight ratio DR which they proved to be more accurate than workplace illuminance and external illuminance. The days chosen for the simulation were $21^{\text {st }}$ March, $21^{\text {st }}$ June and 22nd December which represent the typical hottest, average and coolest months of the year [8]. Simulations were done for the specific times at $0900 \mathrm{hrs}$,
$1200 \mathrm{hrs}$ and $1800 \mathrm{hrs}$ which represented morning, noon and evening. The daylight performance of the atrium buildings is measured in terms of illuminance, DF and average daylight factor (ADF).

The simulation study was conducted in four phases (Fig. 3). The first phase was a simulation study of a base case model (SET A) with the exact specification as the scale model used in the field experiment. This base case model was tested with absolute illuminance (no roof) under overcast sky conditions. In this case, the illuminance at the centre of the horizontal atrium floor was recorded. In the second phase (SET B), simulations were conducted for the three types of roof configurations and the third phase simulations were done for same roof configurations but with different surface reflectances (SET C). Final phase (SET D) simulations ran for annual impact with complication added to the test. For phase two and three, results of illuminances were recorded at three positions on the horizontal floor namely the centre, central edges and corners.

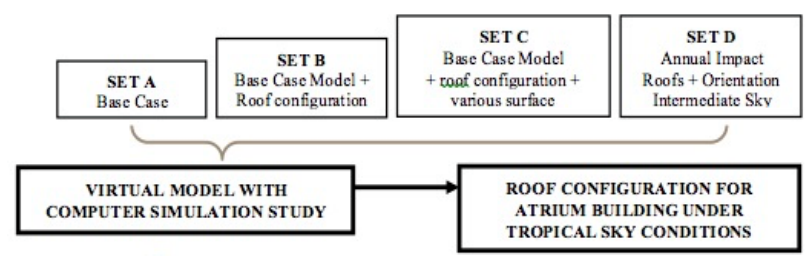

Fig. 3. Four phases with four sets of testing on configurations with computer simulation

\section{Results}

With IESRadiance simulation, the intermediate sky conditions revealed a complex pattern of daylight performance in the atrium installed with three types of roof structures. This intermediate sky for tropics region like Malaysia is having high oktas value which indicates heavy cloud cover. It means that during the daytime, the Malaysian sky is normally bright as the sky is illuminated by clouds. However, heavy cloud cover also hinders radiation heat transfer between objects on the ground with the sky. The major challenges of applying top-fenestration atrium buildings in a tropical region are choosing the right roof fenestration system and reducing the lighting contrast in the atrium spaces. The surface reflectance plays a major role in determining the lighting criteria on the atrium floor.

\subsection{Daylight performance of atrium roofs under overcast skies}

IESRadiance's already established its capability in predicting the levels of daylight illuminance overcast sky conditions for all climate regions [9], as well as for atrium buildings in tropical regions. Hence, the results for this horizontal top-lit atrium under overcast sky conditions were also verified its ability in the virtual investigation. The impact of atrium space configurations on interior illuminance levels was first examined using the Base Case Daylight Factor (BCDF) values of the 
unobstructed atrium model at all three points (horizontal atrium floor), and the results were tabulated in Fig. 4.

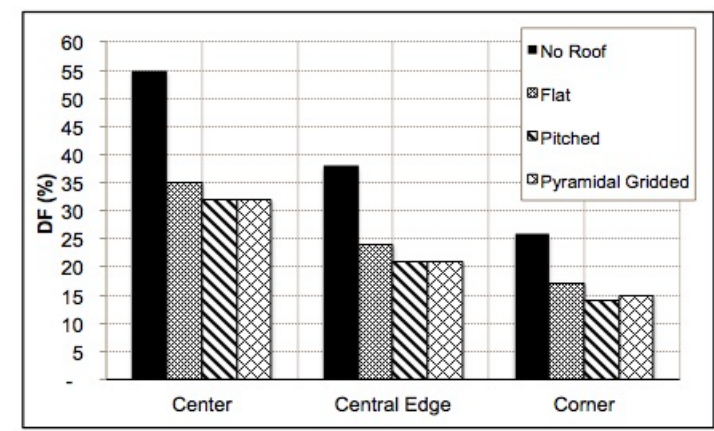

Fig. 4. Computed DF with Absolute Illuminance of Atrium Building with Obstruction under Overcast Sky

For all roof systems, the measurement of DF always shows a decrement for all three positions. The centre atrium is having the highest value, followed by the central edge and corner positions. The light transmittance shows such dynamic patterns for all atrium models. For instance, all three atrium spaces' positions, the cut-off lights were only one-quarter with roof obstructions under the simulation, while there were more than half with the real condition results [2]. Meanwhile, the corner position was constantly most affected by the roof obstructions at all orientations, which brought its DF down to a more acceptable level for the human eye.

The atrium surface reflectance values have a large impact on the DF on the atrium floor. A deeper understanding of the geometric relationship between the sun and the atrium well with roof structures was developed. The proportions of shadow areas and exposed areas on interior surfaces, including the atrium walls and floor were determined by showing the configuration factors of high-luminance interior surfaces when fully exposed to the sun.

Fig. 5 - 6 shows the effects of atrium walls coated with low to high reflectance attached to an unobstructed atrium. Basically, atrium surfaces with $75 \%$ reflectance had an increase of DF by an average of $45 \%$ for all roofs. When the surface reflectance was increased from $25 \%$ to $75 \%$, the DF increased by $10 \%$ at the center, $15 \%$ at the central edge and $20 \%$ at the corner position of the atrium floor. Atrium with low reflectance $(2 \%$ reflectance) and medium reflectance (25\% to $50 \%$ reflectance) saw a higher drop in DFs with the structured atrium roof compared to an atrium left unobstructed. While the high reflectance atrium ( $75 \%$ reflectance) saw a steadier drop in the DFs with more complex roof structures. The results not only showed that the atrium with and without roof obstructions had up to $20 \%$ DF discrepancies but also confirmed that the inclusion of roof structures and complexity of inclination reduced the DF significantly at lower reflectance surfaces (up to 58\% reduction).

The complexity and inclination of roof structures also increase the atrium wall reflectance values for a given atrium roof type with the increment of DF on the floor (Fig. 6).

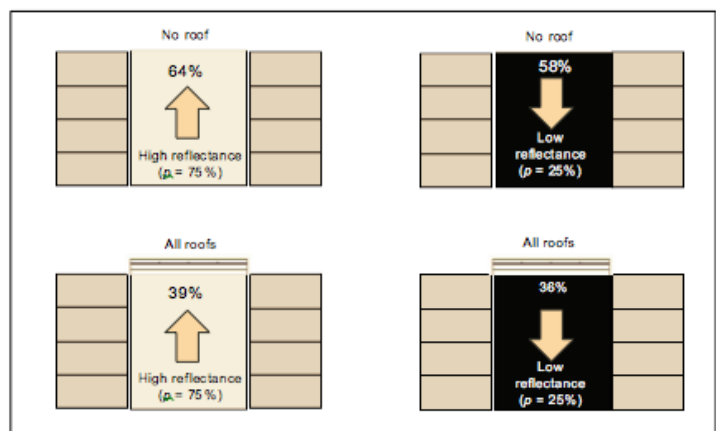

Fig. 5. Summarised findings of Low to High Surface Reflectances Atrium Surface (Wall and Floor) Coated computed with and without Roof Obstruction at the Centre of the Atrium Floor

A high reflectance wall surface of the atrium can improve DF in the centre position of an atrium but may not necessarily improve DFs across the entire floor, due to the that limited the lights to be splayed. Particularly if there are darker surfaces immediately adjacent to the atrium floor.

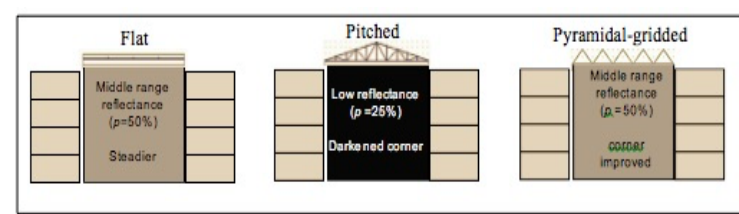

Fig. 6. The Effect of Various Range Surface Reflectances With Inclusion of Different Roof Types at Atrium Floor

\subsection{Daylight performance of atrium roofs under intermediate skies}

The application of roof obstructions on the horizontal aperture of the atrium roof was again tested under intermediate skies (with sun) mode to verify the daylighting performance. From the daylight transmittance magnitudes and distributions, the results showed varied impacts in terms of illuminance values. The uniqueness of Malaysia that falls under tropical climates is her sky. The Malaysian sky has been identified as the intermediate sky (i.e. average cloudy condition with cloud cover value of 6-7 oktas).

It is apparent for both the flat and pitched roofs that the highest illuminations at noon were at the centre location for all three seasons and the highest lux values were recorded for all three locations of atrium floor. In fact, they were also the highest lux values of the day. Interestingly, this situation did not happen for the pyramidal-gridded roof where the highest lux at noon was not at its centre location. For the flat and pitched roofs, for the central-edge position, the west side dominates with the highest lux early in the year, and then at mid-year, it moved from south to east. Then, later in the year, the north side dominates.

From Fig. 7, it shows throughout the year, the atrium with the flat roof appeared as the steadiest roof configuration in terms of letting in daylight. The daylight amount being utilised above the maximum limit only occurred at the centre and towards the edge of the atrium floor. Apparently, this condition assures the capacity of 
human adaptation to light intensity and involves less complicated techniques to direct and utilise light into the adjacent or overlooking spaces around the atrium.

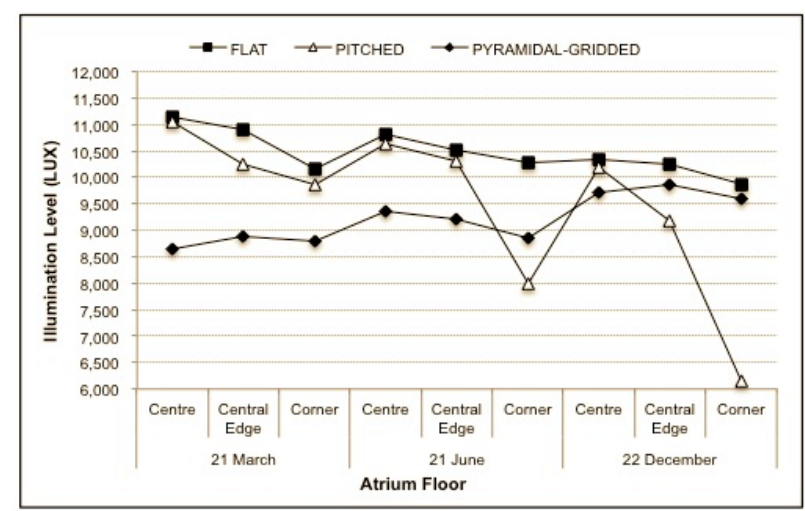

Fig. 7. Illuminance Values against Atrium Roof Obstruction Type at Various Floor Positions Simulated Under Intermediate Sky (With Sun) at $1200 \mathrm{hrs}$ on 21 March, 21 June and 22 December.

The pitched roof is perhaps the least preferred type of atrium roof for atrium buildings. Daylight transmitted into the atrium space is mostly beyond the maximum utilisation level. This leads to a challenging approach when dealing with internal conditions being exposed to longer durations of very high illuminance levels from noon till evening. Further, the evening conditions became more difficult as high intensities of light were captured at all parts of the atrium. However, attention shall be given to the pyramidal-gridded roof. The daylight performance with this roof configuration was slightly improved than a pitched roof. Although the daylight level was beyond the maximum limit of utilised illuminance, the overall illuminance received at the atrium floor was lower than the pitched roof.

\subsection{Atrium parametric strategies for improved daylight performance under Malaysian sky conditions}

The simulation investigation allowed for further evaluations on the daylight penetration levels in the atrium, while the application of various modern structured roof constructions for the atrium allowed better insight into their effects upon the variations of daylight distribution patterns in terms of DF on horizontal surfaces.

Firstly, the roof obstructions affect the distribution of daylight in the atrium well with reduced the light transmittance when the glazing bars were viewed more obliquely. For a four-sided atrium, this usually means a reduction of light on the upper floors. This top-lit transmittance roof obstruction actually plays a major role where it either limit, reflects or splays daylight away from the illuminances to the corners and works efficiently with $50 \%$ or higher solid wall transmittance. Apparently, it also produces useful daylighting contributions to its parent building under diffused skies. The flat roof had the highest daylight illumination for both sunny and overcast sky conditions. Contrast is often desired in the atrium to contribute to the drama and aesthetic value of such spaces. Recommendation pertaining to the optimum combination of components for the entire atrium perimeter cannot be merely stated, excluding other architectural priorities such as glare, functional requirements and so on. This basically will determine the overall transmittance as well as the configurations of its walls, whether uniform or light-andshadow play. This should also determine the desired configurations of the top-fenestration and the amount of solid area on their walls and its reflectance. It also found that under overcast skies, critical attention should be made to the corners of the atrium floor especially for north- and west-facing atrium surfaces as the illuminance values decreased sharply for all types of roofs.

Secondly, the roof obstruction versus atrium shape. It was observed that the influence on transmission losses is small as the area of roof obstruction is similar to the atrium roof shape. The conflicts with the daylight penetration levels at the corner of the atrium floor where the roof structures also function to splay light to the corners. This is where the flat atrium roof is preferable as it appears to have a more stable distribution when orientations become critical. The illuminance values are directly proportional to the spacing of the obstructions. For higher roof inclinations, the value is inversely proportional.

Thirdly, roof obstruction versus atrium well reflectance. More complex roof configurations produced uneven daylight distribution on the higher reflectance values. Atrium skylight roofs with no inclination or simpler roof configurations such as the flat roof performed better for lower surface reflectance ranges. While complicated roof forms such as the pyramidalgridded atrium roof did not reflect nor splay more lights to the corners of the atrium floor but obtained better daylight contribution only at higher surface reflectance. The structured flat roof is better than the middle range of the other two roofs even at the lower range of surface reflectance. The variation of reflectance values appeared to improve the daylight distribution at the atrium floor corners, making it steadier. This would eventually increase the uniformity of light distribution on the atrium floor.

\section{Conclusion}

Fig. 8 summarised the design choices of the physical parameters of atrium roof which can be concluded as the data and design guides for atrium building with reference to tropical sky conditions.

The criteria are summarised above concluded that roof configuration which determines by the roof structure and orientation of roof aperture had the most profound impact on atrium daylighting and sunlighting in tropical atrium buildings, thus resulting in a unique pattern of atrium daylight performance under tropical sky conditions. 


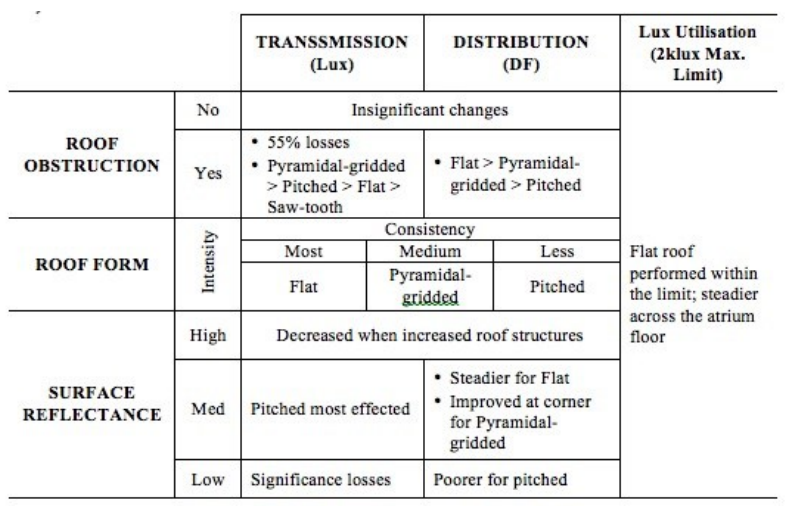

Fig. 8. Daylight Performances of Atrium Buildings for Different Roof Configuration Under Malaysia Sky Conditions

\section{References}

1. J. Yunus, S. S. Ahmad, and A. Zain-Ahmed, "Harnessing Daylight in Atrium: Parametric Influences on the Daylight Conditions at the Atrium Floor," Advanced Science Letters, vol. 22, no. 5-6, pp. 1161-1165, 2016.

2. J. Yunus, "Roof Configurations For Daylight Performances In Malaysian Atrium Buildings Under Tropical Sky Conditions," PhD, Faculty of Architecture, Planning and Surveying, Universiti Teknologi MARA, 2017.

3. J. Yunus, S. S. Ahmad, and A. Zain-Ahmed, "Analysis of Atrium's Architectural Aspects in Office Building under Tropical Sky Conditions," in International Conference In Social and Science Research (CSSR2010), Kuala Lumpur, 2010: IEEE.

4. S. L. Kuan, "Daylighting of Atria in Singapore," Master of Arts (Architecture), Department of Architecture, National University of Singapore, Singapore, 2006.

5. M. H. Ahmad and M. T. Mohamad Rasdi, Design Principles of Atrium Buildings for the Tropics. Johor: Universiti Teknologi Malaysia, 2000.

6. A. R. Othman, " The use of PV to replace typical skylight and its energy generation for cooling and lighting in Malaysian atria " Ph.D., Faculty of Architecture, Planning and Surveying, University Teknologi MARA, 18318, 2013.

7. M. Mousavi, T. H. Khan, and Y.-W. Lim, "Empirical Validation of Radiance-IES Daylight Simulation for Furnished and Unfurnished Rooms under Tropical sky," International Journal of Sustainable Building Technology and Urban Development, vol. 7(1), pp. 1-9, 2016.

8. A. Thanachareonkit, "Comparing Physical and Virtual Methods for Daylight Performance Modelling Including Complex Fenestration Systems," PhD, Solar Energy and Building Physics Laboratory (LESO-PB), Ecole Polytechnique Federale de Lausanne, Lausanne, Switzerland, 2008.

9. M. Ghasemi, M. Z. Kandar, M. Noroozi, F. Yazdipur, S. Namaziyan, and M. Roshan, "Capability of Computer Simulation Software for Predicting Average Daylight Factors in a Vertical
Top-Light Atrium," Journal of Basic and Applied Scientific Research, vol. 3, no. 11, pp. 96-105, 2013. 